\title{
A NOVEL ADDRESSABLE SWITCHING MICRO CORNER CUBE ARRAY FOR FREE-SPACE OPTICAL APPLICATIONS
}

\author{
C.-S. Chang, T.-S. Chu, L.-S Huang, C.-Y. Chang, S.-Y Zeng, M.-H. Wen, Y.-K Yen \\ Institute of Applied Mechanics, National Taiwan University, Taipei, Taiwan
}

Telephone: 886-2-3366-5653 Fax: 866-2-3366-5639 E-mail: lshuang@mems.iam.ntu.edu.tw

\begin{abstract}
We report on a novel addressable switching micro corner cube array for wide free-space optical applications. The corner cube, composed of three mutually orthogonal reflective surfaces, may reflect incident light beam at any angle to its incoming direction. The use of the reflective corner cube array is equivalent to an integration of a lens and a well-aligned mirror array in an appropriate optical configuration. Critical alignment problems can be greatly alleviated. Incorporated with a grating and other optical components, the corner cube is preset to maintain reflective light to its incoming direction, or allowed to provide actuation on one of the reflective planes, causing destruction of its three mutually orthogonal planes for free-space wavelength selection or intensity variation.
\end{abstract}

\section{INTRODUCTION}

In the recent years, micro optics has gradually been playing an important role in optical communication, imaging and instrumentation. A corner cube, composed of three orthogonal surfaces as shown in Fig. 1, can be used to reflect incident light back to its incoming direction [1]. By setting up with a grating and other optical components, the addressable control of comer cube array is able to regulate the wavelength of the reflective light [2]. In this report an array of micromachined corner mirrors is first reported to manipulate light beam in free space. Through MEMS technology, the micro corner cube allows to provide actuation on one of the reflective planes, causing destruction of preset orthogonal planes. The concept is illustrated in Fig. 1(a). As the in-plane mirror is tilted, the light path no more reflects back to its incoming direction.

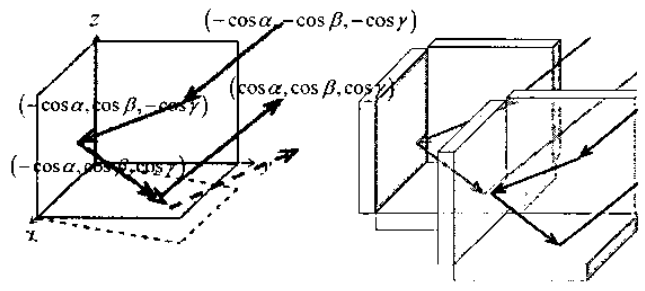

Fig. 1 (a) An illustration of light path switching in one corner cube. (b) A schematic of a corner cube array.
In addition, the use of the corner cube mirrors may not need a lens for collimation in an appropriate optical configuration, thus simplifying the optical system and reducing alignment problems in assembly [2].

\section{THEORY AND DESIGN}

First of all, the corner cube that reflects a light beam back along its incoming direction can be mathematically proved using the ray tracing. As shown in Fig. 1(a), the coordinate is set on the corner cube, and the incoming direction of the light is $(-\cos \alpha$, $-\cos \beta$, $-\cos \gamma$ ), where $\alpha, \beta, \gamma$ are the incident angle between incoming light and the $x, y, z$ axis, respectively. Therefore, the light can be reflected in turn by three planes which are situated on $y z, x z$, and xy plane, respectively. The light path can be demonstrated as

$\left[\begin{array}{ccc}1 & 0 & 0 \\ 0 & 1 & 0 \\ 0 & 0 & -1\end{array}\right]\left[\begin{array}{ccc}1 & 0 & 0 \\ 0 & -1 & 0 \\ 0 & 0 & 1\end{array}\right]\left[\begin{array}{ccc}-1 & 0 & 0 \\ 0 & 1 & 0 \\ 0 & 0 & 1\end{array}\right]\left\{\begin{array}{l}-\cos \alpha \\ -\cos \beta \\ -\cos \gamma\end{array}\right\}=\left\{\begin{array}{l}\cos \alpha \\ \cos \beta \\ \cos \gamma\end{array}\right\}$

The incoming and reflective lights simply shows the opposite signs that indicate an opposite direction for two beam.

In fabrication consideration to achieve both intersected vertical mirror array, surface-micromachined vertical mirror may take tedious work in assembly. This paper reports a bulk-micromachined approach to constitute all the required mirror elements. Fig. 2 illustrates a schematic of a switching micro corner cube. If the incident light angle toward the corner cube at $45^{\circ}$, the optimal size in one set of the comer cube is designed in which the manufacturable in-plane mirror is 700 $\mu \mathrm{m} \times 700 \mu \mathrm{m}$ incorporating the vertical mirror in height of $350 \mu \mathrm{m}$.

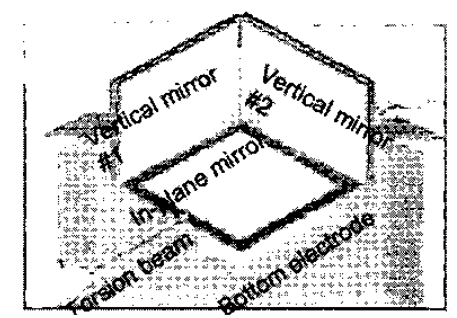

Fig. 2 The schematic of an addressable switching comer cube 


\section{FABRICATION AND ASSEMBLY}

A micro corner cube requires three orthogonal planes with virtually flat and relfective surfaces. Each micromachined corner cube is constituted by three elements: a unit of vertical sideboard mirrors, a movable flat mirror, and a bottom electrode.

Fig. 3 shows the entire fabrication and assembly process flow. All the elements were bulk-micromachined from single crystal silicon material rather than the one in surface micromachining that requires tedious vertical mirror assembly and inherently stress-induced mirror curvature. The large in-plane mirror and a bottom electrode were anisotropically micromachined from Si (100) wafers. A thin gold film was evaporated onto the mirror surfaces and electrodes for reflectivity and conductivity, respectively. The in-plane mirror was then assembled by anodically bonding both elements on the same glass carrier.

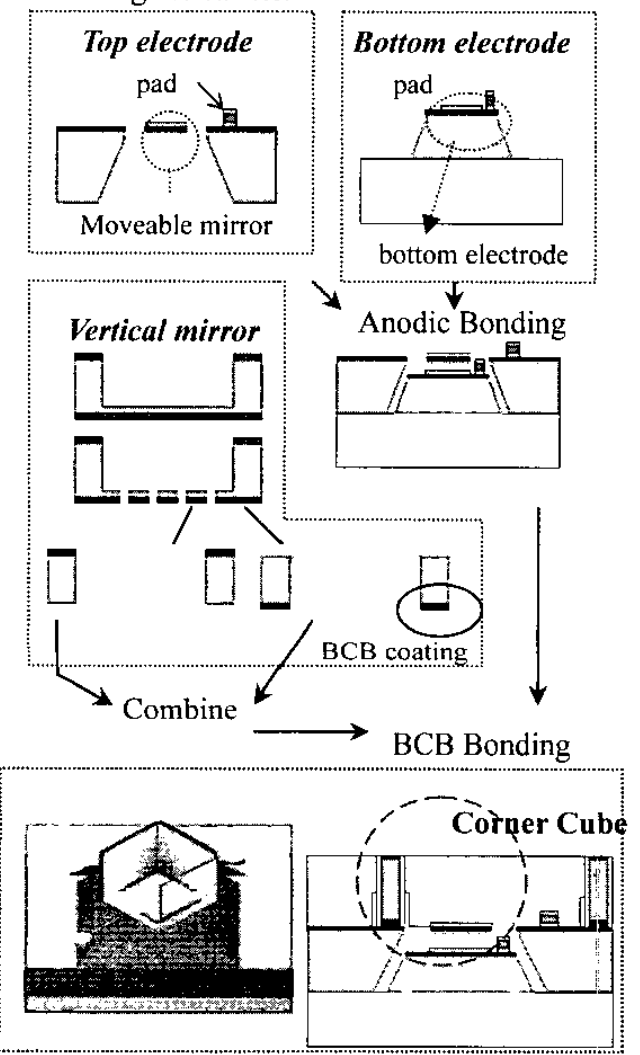

Fig 3. Fabrication and assembly flow of addressable switching corner cube array.

In the fabrication of mutually orthogonal sideboard mirrors, the vertical planes were made with bulk-micromachining in a Si (110) wafer. We then flipped the wafers, and the subsequent deep RIE was used to make lock-in slots in depth of $400 \mu \mathrm{m}$ in the following assembly, which is shown in Fig. 4. The gold film was then deposited for high reflection. With two chips of the vertical mirror arrays having lock-in slots, a matrix of the sideboard mirrors was formed in the assembly of an intersectional arrangement. The concept of the assembly of the sideboard mirrors is shown in Fig. 5.

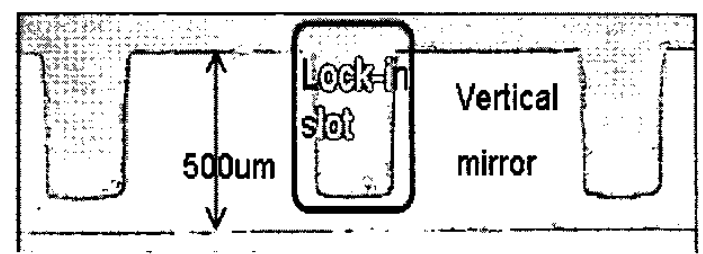

Fig. 4 The side view of a vertical mirror and lock-in slots.

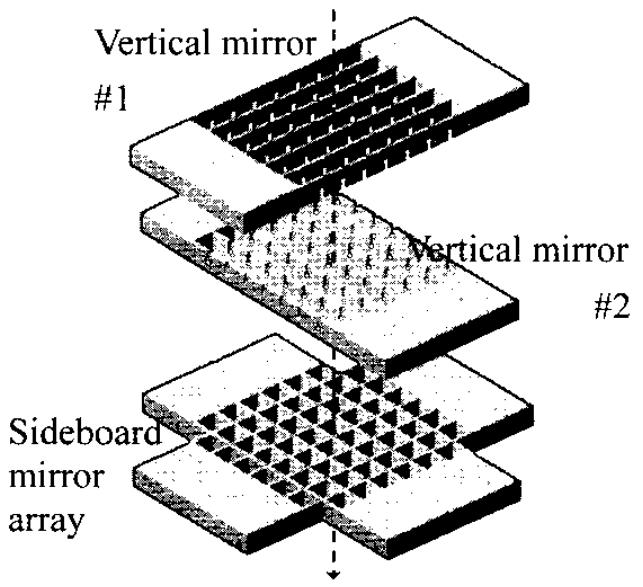

Fig. 5 The exploded diagram of the sideboard mirror array

To constitute the addressable switching corner cube, the patterned polymer Benzocyclobutene (BCB) on the in-plane mirror substrate was heated in joining the fixed vertical sideboard mirror.

\section{EXPERIMENTAL RESULTS}

Fig. 6 shows the SEM picture of the in-plane mirror, the dimension of the flat mirror is $700 \mu \mathrm{m}$ by $700 \mu \mathrm{m}$ with the thickness of $13 \mu \mathrm{m}$, which is rather large compared to the surface-micromachined poly-Si mirtor. In Fig. 7, the SEM picture shows a set of sideboard mirror, which is constituted by two intersectional, erect mirror arrays. Each ridge thickness of the vertical mirror is $150 \mu \mathrm{m}$, and the area of the sideboard mirror surround is $700 \mu \mathrm{m} \times 700$ $\mu \mathrm{m}$, which corresponds to the area in one in-plane mirror. After the assembly of the erect sideboard mirror and in-plane mirror, the micro corner cube array was achieved finally. Fig. 8 demonstrates a photographic picture of the addressable switching corner mirror array.

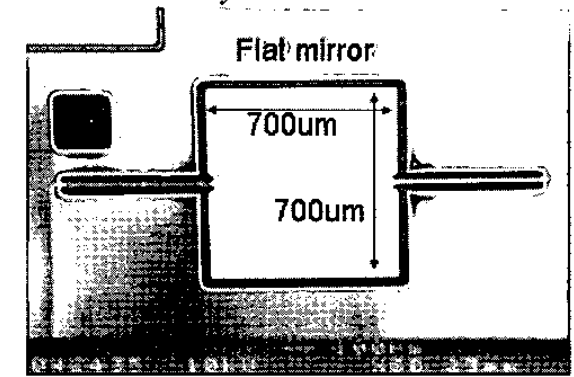

Fig. 6 The SEM of the moveable flat mirror 


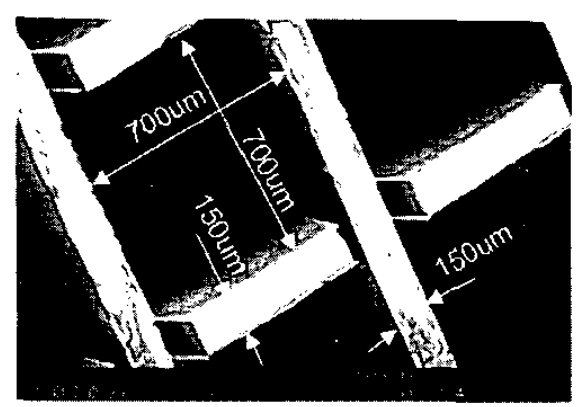

Fig. 7 The close-up SEM of the sideboard mirror

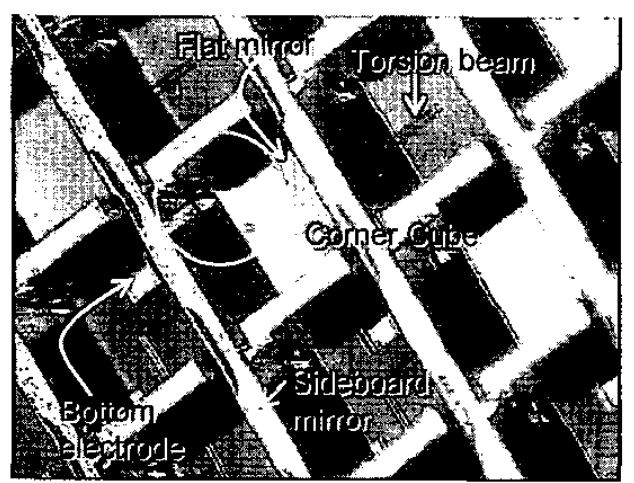

Fig. 8 The photographic picture of the addressable switching corner cube array.

Characterization of the switching micro corner cube has been conducted in mechanical and optical properties to provide in-depth understanding for its applications. In its mechanical characteristics, static actuation, dynamic resonance, and switching time have been characterized, respectively. As for the optical properties, the mirror curvature and verticality of the mirror array are also conducted and discussed.

\section{Mechanically static and dynamic characterization}

Fig. 9 demonstrates an in-plane mirror actuated behavior. The static mirror titling with respect to varied electric voltages may adjust an output optical intensity. When the in-plane mirror angle is tilted at approximate $1.4^{\circ}$, the device reaches its abrupt pull-in condition at 110 volt. In addition, the mechanical resonance frequency was measured to be $2.9 \mathrm{KHz}$.

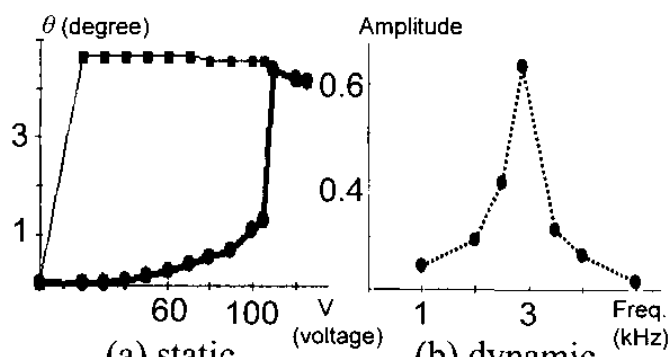

(a) static

(b) dynamic

Fig. 9 The static and dynamic behavior of the actuated in-plane mirror.

Fig. 10 shows the optical experiment for measuring the micro corner cube switching. The switchable corner cube lies at the light path reflected from a mirror after the emitted light passes through the NPBS first. The signal can be detected from the incoming light from the non-polarized beam splitter (NPBS).

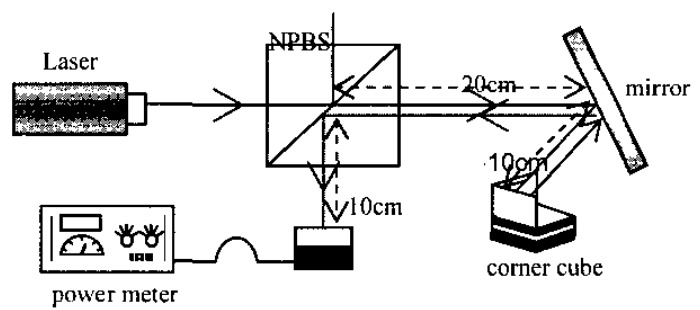

Fig. 10 The measurement system for the micro comer cube switching.

Fig. 11 (a)(b) shows the optical switching time of the corner cube actuated in a 100 volt. It is found that the fall time is approximate $1.5 \mathrm{~ms}$, and the rise time is approximate $2 \mathrm{~ms}$.
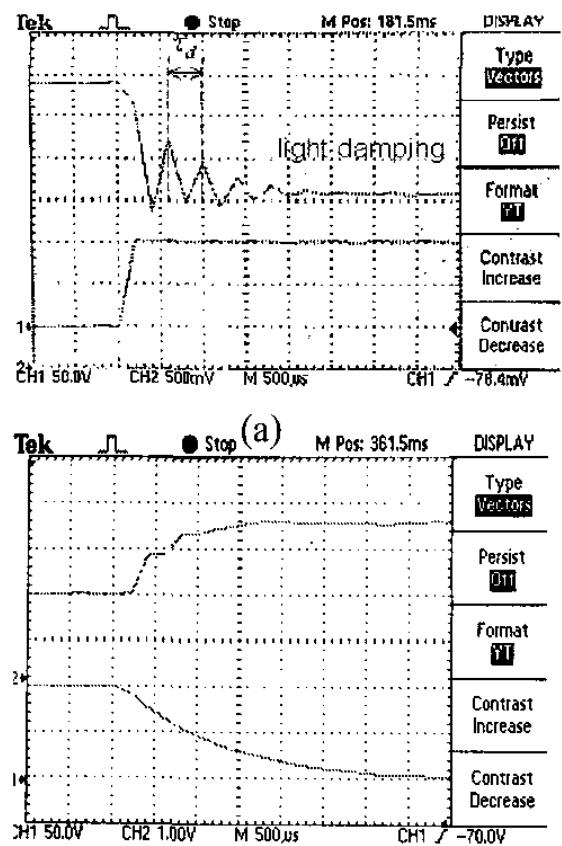

(b)

Fig. 11 The spectrum of the device switching time and its associated voltage applications (a) Fall time $(1.5 \mathrm{~ms})$ with respect to 100 volt application. (b) Rise time $(1.25 \mathrm{~ms})$ at the voltage release.

\section{Optical properties}

The roughness and the flatness were inspected through the atomic force microscope (AFM) and instrumental interferometer, WYKO MHT III. In the roughness of both virtually orthogonal planes, the vertical and horizontal surfaces were measured in root-mean-square (RMS) value to be $3.32 \mathrm{~nm}$ and $4.18 \mathrm{~nm}$, respectively, which demonstrates insignificant surface scattering. As for the flatness, 
the in-plane mirror of the thickness of $12 \mu \mathrm{m}$ in thickness and $700 \mu \mathrm{m} \times 700 \mu \mathrm{m}$ in mirror size is more even critical in radius of curvature than the $150 \mu \mathrm{m}$ thick vertical mirror. As shown in Fig. 9, the radius of curvature was measured to be $34.31 \mathrm{~mm}$ by interferometer.

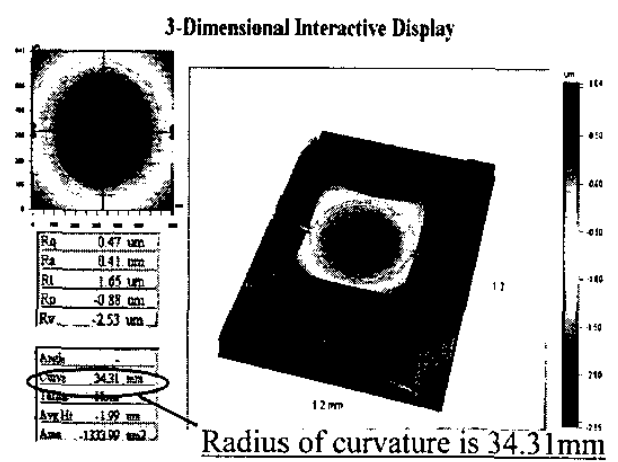

Fig. 12 The surface profile image of a horizontal mirror

As for verticality between mirrors, Fig. 10 demonstrates a simple means to characterize the deviation out of a right angle. As a laser light beam is emitted to one set of two virtually vertical mirrors, two reflective light beams are found back to its incoming direction due to an angle deviation out of $90^{\circ}$. With an aid of a ray tracing software, at a given distance of a back screen the deviation angle can be obtained at a measured distance between two spots. With this means, the vertical mirrors of different planes with respective to its in-plane substrate are found to be around $90^{\circ} \pm 0.6^{\circ}$.

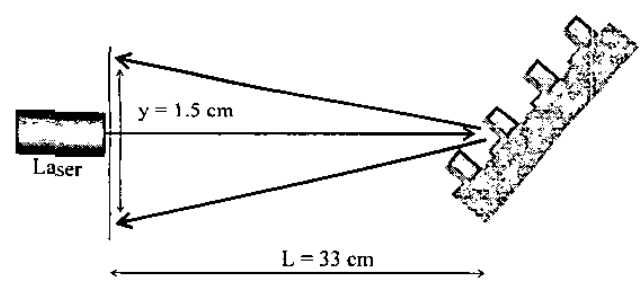

Fig. 13 The measurement of the included angle between sidewall mirror and in-plane mirror.

The verticality between two intersectional, erect mirror arrays in two planes was also considered through the geometric configuration. After the assembly of intersectional mirrors through lock-in slots, the tolerant angle between two intersectional mirror arrays was calculated to be $90^{\circ} \pm 0.33^{\circ}$. Since both intersectional mirror arrays were bulk-micromachined, the uniformity was consistent on each set of corner cubes. The efficient assembly and consistent uniformity greatly benefit from the bulk-micromachining technique.

\section{CONCLUSION}

The new addressable switching micro corner cube array is successfully demonstrated. Characterization of the switching micro corner cube has been conducted in mechanical and optical properties to provide in-depth understanding for its applications. The angle deviation out of 900 between the in-plane and erect mirrors is around 0.60 . Its switching time achieves $1.5 \mathrm{~ms}$ for fall time and $1.25 \mathrm{~ms}$ for rise time. According to the previous work, incorporated with a grating and other optical components, the corner cube is potentially to provide actuation on in-plane mirror, causing destruction of its three mutually orthogonal planes for free-space wavelength selection or intensity variation.

\section{REFERENCE}

[1] L. Zhou, K.S.J. Pister, J.M. Kahn, "Assembled Comer-Cube Retroreflector Quadruplet," IEEE MEMS, Las Vegas, Nevada, pp. 556-559, Jan., 2002.

[2] T.-S. Chu, L.-S Huang, C.-Y. Chang, C.-S. Chang, W.-F. Ye, M.-H. Wen, C.-C.Yang, "A New Addressable Corner Micromirror Array for Free-Space Optical Applications", IEEE/LEOS Optical MEMS, Lugano, Swizerland, Aug. 2002. 